\title{
Radiative Thermostat Driven by the Combined Dynamics of Electrons, Phonons and Photons
}

\author{
Jose Ordonez-Miranda ${ }^{1, *}$ \\ ${ }^{1}$ Institut Pprime, CNRS, Université de Poitiers, ISAE-ENSMA, F-86962 Futuroscope Chasseneuil, France
}

(Dated: August 9, 2020)

\begin{abstract}
Based on the metal-insulator transition of two phase-change materials (PCMs) with asymmetric thermal conductivities and emissivities sensitive to temperature, a radiative thermostat capable of maintaining a constant temperature without consuming energy is proposed. This is done by deriving explicit expressions for the heat flux and temperature profiles of the thermostat surrounded by these PCMs and demonstrating that it can be maintained at a temperature nearly equal to the common transition temperature of both PCMs, as the environmental one changes more than $20 \mathrm{~K}$. It it shown that the combined operation of two PCMs is much better than non-PCMs to develop energyfree termostats, specially those supporting poor thermal conduction or dominant heat radiation. The obtained results thus establish a fundamental theoretical method to exploit the nonlinear heat transport driven by electrons, phonons and photons, for saving energy on temperature preservation and thermal insulation.
\end{abstract}

PACS numbers: 65.60.+a; 65.80.-g; 65.90.+i

\section{INTRODUCTION}

Heat is one of the main forms of wasted energy and its control is hence of critical importance to efficiently manage the energy resources of nature, which is under increasing stress due to the growing energy-hungry world population and global warming issues. The management of heat transport has become very challenging, as revealed by, for instance, the high percentage $(68.5 \%)$ of energy lost in developed countries as waste heat that is mainly released into the environment [1]. Around $15 \%$ of the remaining energy is used for thermal insulation or preserving the temperature of industrial fields, buildings, homes, etc. [2]. These representative figures show that the reduction of the energy consumption is a huge scientific, technological and societal challenge in the energy sector, that has been partially addressed through the nonlinear heat transport.

Strongly nonlinear heat conduction (radiation) shows up in materials with temperature dependent thermal conductivities (emissivities), whose tailoring allows modulating heat currents [3]. Over the past two decades, many research groups have been exploiting these thermally driven properties to control the heat transport via phonons, electrons, and photons [4-12]. Several conceptual devices, such as thermal diodes [1318], thermal transistors [19-23], thermal memories [24] and thermal memristors [25, 26] were proposed; and the working principles of some of them were experimentally demonstrated[27-29]. Furthermore, based on two phase change materials (PCMs) with asymmetric thermal conductivities, Shen et al. [30] developed a temperaturetrapping theory and proposed an energy-free thermostat able to self-maintain a desired constant temperature

* jose.ordonez@cnrs.pprime.fr without consuming energy, regardless of the sizeable changes of its environmental temperature. More recently, Wang et al. [31] introduced thermoelectric effects in this latter theory and proposed a negative-energy thermostat capable of generating electricity with energyfree maintenance of a constant ambient temperature. These two latter works $[30,31]$ were also applied to significantly improve the performance of thermal and thermoelectric cloaks, whose central regions are kept at pretty much constant temperatures, as the ambient ones vary in relatively large intervals. The operation of these two thermostats and cloaks is based on conductive heat currents and therefore their performances are limited by the inevitable presence of interface thermal resistances between the involved PCMs. This limitation could, however, be overcome by a radiative thermostat supporting photon heat currents between their contactless terminals, as is the case of nanophotonic thermostats based on multilayer structures and operating with solar radiation [32].

The purpose of this work is to demonstrate the proof of principle of a radiative thermostat, a cavity able to self-maintain a constant temperature despite being exposed to sizeable changes of its environmental temperature. This is achieved by using two PCMs as the cavity walls, whose asymmetric thermal conductivities and emissivities significantly change with temperature, within a narrow interval of temperatures. The proposed thermostat is thus a cavity that capitalizes on the non-linear heat transport driven by both conductive and radiative heat currents, and therefore its working principle is different than the conductive thermostat [30, 31], passive radiative one [32], and other devices of temperature regulation [33-35] consisting of a layered system with a surface emitting radiation to the outer space through the atmosphere's transparency windows in the mid-infrared. The cavity could thus be applied as a constant temperature environment of objects. 


\section{THEORETICAL MODELING}

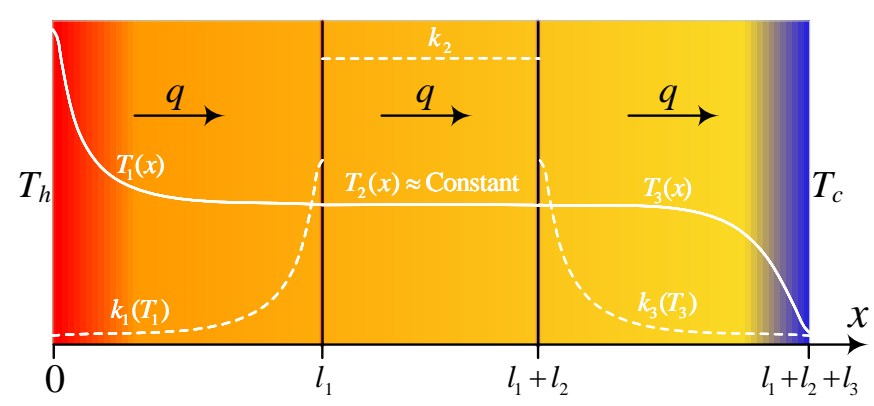

FIG. 1: Scheme of a cavity with a nearly constant temperature maintained by two PCMs. The solid line stands for the temperature profile generated by the temperature dependence of the asymmetric thermal conductivities (dashed lines) of the PCMs. The surfaces $x=0$ and $x=l_{1}+l_{2}+l_{3}$ are set at the steady-state temperatures $T_{h}$ and $T_{c}\left(<T_{h}\right)$, respectively.

Let us consider two PCMs exchanging heat by radiation and conduction through a cavity, as a result of the temperature difference $T_{h}-T_{c}>0$ of their external surfaces, as shown in Fig. 1. In order to minimize the impact of the enviromental temperature variations on the cavity temperature profile, i consider that the left-hand (right-hand) side PCM behaves as a thermal insulator at the relatively high (low) temperature $T_{h}$ $\left(T_{c}\right)$, while it becomes a thermal conductor for lower (higher) temperatures. According to the Fourier's law of heat conduction, these conditions can be achieved when the thermal conductivity $\left(k_{1}\right)$ of the left-hand side PCM increases as the temperature decreases, while the one $\left(k_{3}\right)$ of the right-hand side PCM exhibits the opposite behavior, as depicted in Fig. 1 through the dashed lines. More explicitly, this law along with the StefanBoltzmann's one and the principle of energy conservation establish that the steady-state heat flux $q$ propagating along the three layers is given by [36]

$q=-k_{1}\left(T_{1}\right) \frac{d T_{1}}{d x}=\frac{\theta-\psi}{\rho_{2}}+\sigma \varepsilon\left(\theta^{4}-\psi^{4}\right)=-k_{3}\left(T_{3}\right) \frac{d T_{3}}{d x}$,

where $T_{1}$ and $T_{3}$ are the respective temperatures within the PCM1 $\left(0 \leq x \leq l_{1}\right)$ and PCM3 $\left(l_{1} \leq x \leq\right.$ $\left.l_{1}+l_{2}\right), \theta=T_{1}\left(l_{l}\right), \psi=T_{3}\left(l_{l}+l_{2}\right), \sigma$ is the StefanBoltzmann constant, $\varepsilon=\left(\varepsilon_{1}(\theta)^{-1}+\varepsilon_{3}(\psi)^{-1}-1\right)^{-1}$ is the effective emissivity and the thermal resistance $\rho_{2}=l_{2} / k_{2}+2 / h$, with $\varepsilon_{1}(\theta)$ and $\varepsilon_{3}(\psi)$ being the respective emissivities of the facing walls of PCM1 and PCM3, which exchange heat by radiation, conduction and convection through the intracavity gas of thermal conductivity $k_{2}$ and convective heat transfer coefficient $h$. For air, $k_{2}=25 \mathrm{mWm}^{-1} \mathrm{~K}^{-1}$ and $h=5 \mathrm{Wm}^{-2} \mathrm{~K}^{-1}$, within a wide range of temperatures [37]. The effective emissivity $\varepsilon$ driving the intracavity radiation is defined by the Stefan-Boltzmann's law [36] and its values are independent of the optical properties of the intracavity gas, because this gas is assumed to be transparent to the infrared radiation exchanged by PCM1 and PCM3, as is the case of air for temperatures around the room temperature considered in this work [32]. Given that $q$ is a constant, Eq. (2) establishes that $-d T_{n} / d x$ decreases as $k_{n}\left(T_{n}\right)(n=1$ and 3$)$ increases and vice versa, as depicted in Fig. 1.

Considering that both PCMs have a metal-insulator transition at the temperature $T_{0}$, the required temperature dependence of their thermal conductivities $k_{n}$ and mean emissivities $\varepsilon_{n}$, for $n=1$ and 3 , can be described as follows

$$
\begin{aligned}
& k_{n}(T)=k_{l n}+\frac{k_{h n}-k_{l n}}{1+e^{-\beta\left(T-T_{0}\right)}}, \\
& \varepsilon_{n}(T)=\varepsilon_{l n}+\frac{\varepsilon_{h n}-\varepsilon_{l n}}{1+e^{-\beta\left(T-T_{0}\right)}},
\end{aligned}
$$

where $k_{l n}\left(\varepsilon_{l n}\right)$ and $k_{h n}\left(\varepsilon_{h n}\right)$ are the thermal conductivities (emissivities) at low $\left(T \ll T_{0}\right)$ and high $\left(T \gg T_{0}\right)$ temperatures, respectively; and $\beta$ is the phasetransition slope of $k_{n}(T)$ and $\varepsilon_{n}(T)$ at $T=T_{0}$. In order to fulfill the external thermal insulation condition depicted in Fig. 1, i consider that $k_{l 1}>k_{h 1}$ and $k_{l 3}<k_{h 3}$, which along with Eq.(2a) determines the behaviors of $k_{1}(T)$ and $k_{3}(T)$ shown in Fig. 2(a). Note that the PCM1 at low temperature $\left(T \ll T_{0}\right)$ is in its metallic phase, which switches to an insulating one at high temperature $\left(T \gg T_{0}\right)$. Taking into account that metals usually exibit emissitities lower than insulators, i consider that $\varepsilon_{l 1}<\varepsilon_{h 1}$, as shown in Fig. 2(b). Therefore, the PCM1 emissivity, as the thermal conductivity $k_{1}$, is bounded between its values for the metallic and insulating phases, such that the ratio $\varepsilon_{h 1} / \varepsilon_{l 1}>1$ allows enhancing (reducing) the radiative heat flux with PCM1 in its insulating (metallic) phase, as established by Eq. (1). For the emissitivy of PCM3, on the other hand, i take $\varepsilon_{l 3}>\varepsilon_{h 3}$ (Fig. 2(b)), given that $k_{3}(T)$ has the opposite behavior of $k_{1}(T)$.

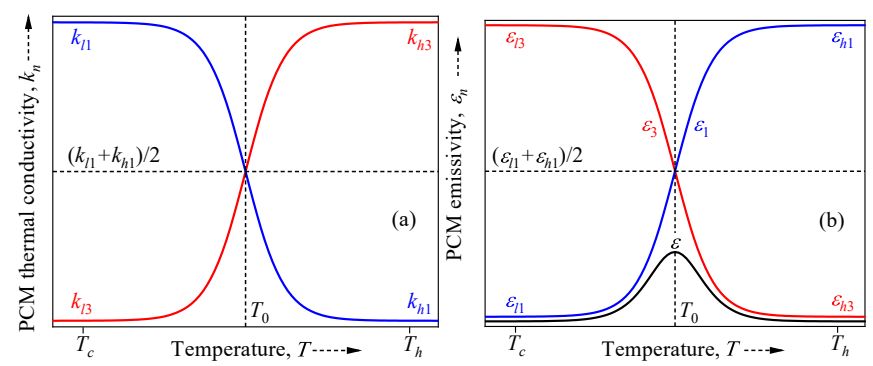

FIG. 2: Temperature dependence of the (a) thermal conductivities and (b) mean emissivities of the two

PCMs involved in Fig. 1. The black line in (b) stands for the effective emissivity $\varepsilon$ defined in Eq.(1).

In practice, the temperature dependence of $k_{1}(T)$ and $\varepsilon_{1}(T)$ can be obtained with polyethylene nanofibers [38] and $\mathrm{Ge}_{2} \mathrm{Sb}_{2} \mathrm{Te}_{5}$ [39], while that of $k_{3}(T)$ and 
$\varepsilon_{3}(T)$ is exhibited by $\mathrm{VO}_{2}$ [3, 40-42] and nitinol [43]. The phase transition of these four PCMs is driven by correlated atomic interactions and therefore the values of their thermal and optical properties at low and high temperatures can be tailored through dopings or by varying the deposition parameters, as was reported for the metal-insulation transition of $\mathrm{VO}_{2}$ [44-47]. Furthermore, the temperature variations of $k_{n}(T)$ and $\varepsilon_{n}(T)$, for $n=1$ and 3, can also be achieved by means of alternating layered structures involving strips of shape memory alloys, as detailled by Shen et al. [30] and Wang et al.[31]. This latter multistep approximation method is particularly suitable to make sure that the values of the transition parameters $T_{0}$ and $\beta$ are the same for both materials surrounding the cavity, given that they will be composed by the same shape memory alloy. In general, the thermal and optical properties of these alloys and PCMs vary significantly due to their metal-insulator transition in a relatively narrow interval of temperatures. This phase transition is the reason why the operation of the radiative thermostat (Fig. 1) is driven by the collective dynamics of electrons, phonons, and photons.

The temperature profiles $T_{1}(x)$ and $T_{3}(x)$ along with the heat flux $q$ can be determined by integrating Eq. (1) for the thermal conductivity in Eq. (2a) and the boundary conditions $T_{1}(0)=T_{h}$ and $T_{1}\left(l_{1}\right)=\theta\left(T_{3}\left(l_{1}+\right.\right.$ $\left.l_{2}\right)=\psi$ and $\left.T_{3}\left(l_{1}+l_{2}+l_{3}\right)=T_{c}\right)$. Final results can be written as follows

$$
\begin{gathered}
q=\frac{k_{h 1}-k_{l 1}}{\beta_{1} l_{1}} \ln \left[\frac{f_{1}\left(T_{h}\right)}{f_{1}(\theta)}\right]=\frac{k_{h 3}-k_{l 3}}{\beta_{3} l_{3}} \ln \left[\frac{f_{3}(\psi)}{f_{3}\left(T_{c}\right)}\right] \\
=\frac{\theta-\psi}{\rho_{2}}+\sigma \varepsilon\left(\theta^{4}-\psi^{4}\right) \\
f_{1}\left(T_{1}(x)\right)=\left[f_{1}\left(T_{h}\right)\right]^{1-x / l_{1}}\left[f_{1}(\theta)\right]^{x / l_{1}}, \\
f_{3}\left(T_{3}(x)\right)=\left[f_{3}(\psi)\right]^{1-\left(x-l_{1}-l_{2}\right) / l_{3}}\left[f_{3}\left(T_{c}\right)\right]^{\left(x-l_{1}-l_{2}\right) / l_{3}}
\end{gathered}
$$

where $f_{n}(T)=\left[1+Z_{n}(T)\right]\left[Z_{n}(T)\right]^{-\lambda_{n}}, \quad Z_{n}(T)=$ $\exp \left[-\beta_{n}\left(T-T_{0}\right)\right]$ and $\lambda_{n}=k_{h n} /\left(k_{h n}-k_{l n}\right)$, for $n=1$ and 3. Taking into account that $k_{l 1}>k_{l 1}\left(k_{l 3}<\right.$ $\left.k_{h 1}\right)$, the function $f_{1}(T)\left(f_{3}(T)\right)$ decreases (increases) monotonically as the temperature $T$ rises and therefore the heat flux $q$ in Eq. (3a) only takes positive values, in agreement with the second law of thermodynamics, for $T_{h}>T_{c}$. Given that the heat flux $q$ is a constant, the third equality in Eq. (3a) establishes a tradeoff between the conductive and radiative contributions, such that the latter one strengthens as the conductive thermal resistance $\rho_{2}$ increases. The temperatures $T_{1}(x)$ and $T_{3}(x)$ are determined by the respective numerical solutions of Eqs. (3b) and (3c), after solving the strongly non-linear system of Eqs. (3a), for the temperatures $\theta$ and $\psi$ of the inner walls. In order to capitalize on the full phase transition of the PCMs and derive an approximate solution of Eqs. (3a), the external temperatures $T_{h}$ and $T_{c}$ are chosen in such a way that they fulfill the condition $T_{h} \gg T_{0} \gg T_{c}$. In this way, the cavity is thermally insulated from the external environment and therefore the temperatures $\theta$ and $\psi$ of the inner walls are expected to satisfy the condition $\beta\left|\theta-T_{0}\right| \ll 1$ and $\beta\left|\psi-T_{0}\right| \ll 1$, as graphically shown below (Fig. 3(a)). Under these conditions, Eqs. (3a) become a system of two linear equations for $\theta$ and $\psi$, which yields

$$
\theta-\psi=\frac{2 R_{2}}{R(r+1)}\left[T_{h}-T_{c}+2 \ln (2) \frac{(r-1)}{\beta}\right] .
$$

where $R=R_{1}+R_{2}+R_{3}, R_{n}=l_{n} / \bar{k}_{n}, \bar{k}_{n}=\left(k_{l n}+\right.$ $\left.k_{h n}\right) / 2$, for $n=1$ and $3, R_{2}=\left(\rho_{2}^{-1}+4 \sigma \bar{\varepsilon} T_{0}^{3}\right)^{-1}, \bar{\varepsilon}=$ $\left(\bar{\varepsilon}_{1}^{-1}+\bar{\varepsilon}_{3}^{-1}-1\right)^{-1}, \bar{\varepsilon}_{n}=\left(\varepsilon_{l n}+\varepsilon_{h n}\right) / 2$, and $r=k_{l 1} / k_{h 1}$. For the sake of simplicity and clarity, Eq. (4) was derived by using the condition $k_{l 1}=k_{h 3}$ and $k_{h 1}=k_{l 3}$, which establishes the mirror-like symmetry of $k_{1}(T)$ and $k_{3}(T)$ around the transition temperature $T=T_{0}$. Equation (4) indicates that the temperature difference $\theta-\psi$ can be minimized by reducing the thermal resistance $R_{2}$, enhancing the thermal conductivity contrast $r>1$ and getting steep phase transitions (high $\beta$ values). As these three parameters are determined by the thermal and optical properties of the considered PCMs, the values of $\theta-\psi$ are expected to be much smaller than the corresponding ones obtained with non-PCMs $(\theta-\psi=$ $R_{2}\left(T_{h}-T_{c}\right) / R$ for $\left.r=1\right)$. Note also that the radiative contribution boosts the reduction of $\theta-\psi$ through a high emissivity $\bar{\varepsilon}$ and/or transition temperature $T_{0}$, which only appears due to the intracavity radiative heat transport.

\section{RESULTS AND DISCUSSION}

The thermal performance of the radiative thermostat is now numerically analyzed by means of the temperature profiles for different values of the environmental temperatures and thermal resistance $\rho_{2}$. To show how the cavity temperature could be maintened at room temperature, we choose $T_{0}=300 \mathrm{~K}$ and change the temperature $T_{h}$ through values higher than $T_{0}$ to capitalize on the phase transition of noth PCMs. For the sake of comparison, the temperature $T_{c}=290 \mathrm{~K}$ is kept constant, given that its variations through values $T_{c} \ll T_{0}$ do not change either the thermal conductivity or emissivity of the PCM3, and therefore they will not significantly affect the thermostat temperature profile.

Figures 3(a) and 3(b) show the inner walls' temperatures $\theta$ and $\psi$ along with their difference $\theta-\psi>$ 0 as functions of $T_{h}$, respectively, for three representative values of the thermal resistance $\rho_{2}$. Note that both $\theta$ and $\psi$ increases almost linearly with $T_{h}$, when this temperature is smaller than the transition one $\left(T_{h}<\right.$ $\left.T_{0}\right)$. This behavior is typically generated by nonPCMs and appears due to the fact that the environment temperature $T_{h}$ is too low to excite the phase transition of both PCMs (see Fig. 2). By contrast, for $T_{h}>T_{0}$, the 

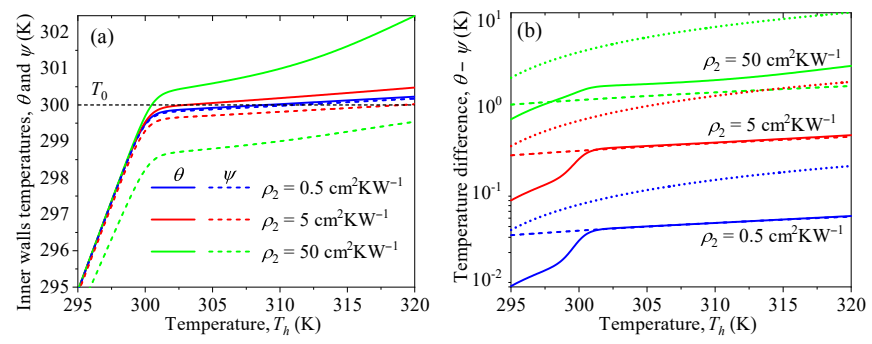

FIG. 3: (a) Inner walls temperatures and (b) their difference as functions of the temperature $T_{h}$.

Calculations were done with $T_{c}=290 \mathrm{~K}, l_{1}=l_{3}=3$ $\mathrm{cm}, T_{0}=300 \mathrm{~K}, \beta=2 \mathrm{~K}^{-1}, k_{l 1}=k_{h 3}=50 \mathrm{Wm}^{-1} \mathrm{~K}^{-1}$, $k_{h 1}=k_{l 3}=1 \mathrm{Wm}^{-1} \mathrm{~K}^{-1}, \varepsilon_{l 1}=\varepsilon_{h 3}=0.2$,

$\varepsilon_{h 1}=\varepsilon_{l 3}=0.8$ and three values of $\rho_{2}$. The solid, dashed, and dotted lines in (b) stand for the respective predictions of Eqs. (3a), (4), and (1). This latter one was obtained for non-PCMs with thermal conductivities $k_{n}=\bar{k}_{n}$ and emissivities $\varepsilon_{n}=\bar{\varepsilon}_{n}$.

slopes of the inner walls' temperatures reduce drastically and their values tend to keep close to $T_{0}$, especially for the two smaller values of $\rho_{2}$. In this case, the temperature difference $\theta-\psi$ is smaller than $1 \mathrm{~K}$ and is well predicted by Eq. (4), as shown by the blue and red dashed lines in Fig. 3(b). This key fact indicates that the temperature of the central material can be maintained pretty much constant and equal to the transition temperature of both PCMs, without the injection of external energy, when the environment temperatures vary through values higher and lower than $T_{0}\left(T_{h}>T_{0}>T_{c}\right)$. In practice, the thermal resistance $\rho_{2}=0.5 \mathrm{~cm}^{2} \mathrm{KW}^{-1}$ could be set with either a $2-\mathrm{cm}$ thick thermostat of copper $\left(l_{2}=\right.$ $2 \mathrm{~cm}$ and $k_{2}=400 \mathrm{Wm}^{-1} \mathrm{~K}^{-1}$ ) or a $1.25 \mu \mathrm{m}$-thick cavity of air $\left(l_{2}=1.25 \mu \mathrm{m}\right.$ and $\left.k_{2}=25 \mathrm{mWm}^{-1} \mathrm{~K}^{-1}\right)$. Thicker air cavities yield a higher thermal resistance $\rho_{2}$, which increases the temperature difference of the inner walls to values higher than $1 \mathrm{~K}$. However, the values of $\theta-\psi$ generated by the combined effect of two PCMs for different values of $\rho_{2}$, are relatively smaller than the corresponding ones obtained with non-PCMs, as shown by the dotted lines in Fig. 3(b). Therefore, the temperature profile of the central material (solid or gas) becomes considerably less sensitive to the environment temperature gradients, when is placed in between two PCMs with asymmetric thermal and optical properties than when it is in the middle of two non-PCMs.

The temperature and thermal conductivity distributions inside the three-layer system are shown in Fig. 4 as functions of position, for a $2 \mathrm{~cm}$-thick central layer of copper. The dashed line represents the temperature profile obtained when the two PCMs are replaced by non-PCMs with thermal conductivities and emissivities independent of temperature. Note that near the outer surfaces $x=0$ and $8 \mathrm{~cm}$, the temperature exhibits a pretty much linear behavior due to the relatively constant and low values of the

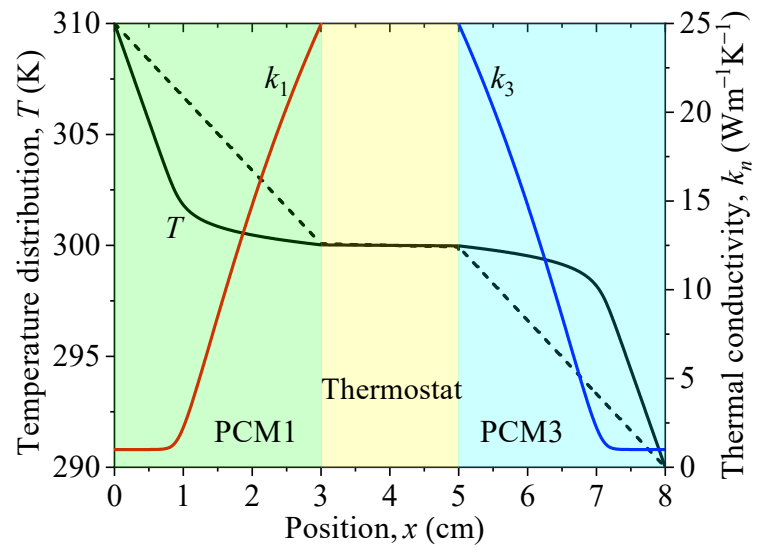

FIG. 4: Temperature and thermal conductivity distributions obtained with Eqs. (3) and (2a) inside the three-layer system, as functions of position, respectively. The dashed line stands for the prediction of Eq. (1) for non-PCMs with thermal conductivities $k_{n}=\bar{k}_{n}$ and emissivities $\varepsilon_{n}=\bar{\varepsilon}_{n}$. Calculations were done for a $\mathrm{Cu}$ central layer with $l_{2}=2 \mathrm{~cm}, \rho_{2}=0.5 \mathrm{~cm}^{2} \mathrm{KW}^{-1}$,

$T_{h}=310 \mathrm{~K}$ and the data in caption of Fig. 3 .

thermal conductivity of both PCMs. For other internal positions, on the other hand, these conductivities change drastically to keep the temperature nearly constant and equal to the transition temperature $T_{0}=300 \mathrm{~K}$, specially within the central layer. This fact is consistent with the one shown in Fig. 3 and is, of course, generated by the combined phase transitions of the two PCMs. Even though the non-linear behavior of temperature within the PCMs is totally different than the linear one (dashed line) obtained with non-PCMs, the temperature values inside the cavity are pretty much the same in both cases. This is reasonable due to the relatively high thermal conductivity of the copper layer and it is expected to change for a cavity with lower (higher) thermal conductivity (thermal resistance $\rho_{2}$ ), as shown in Fig. 5. While the cavity temperature tends to keep close to $T_{0}=300 \mathrm{~K}$, the corresponding one obtained with non-PCMs significantly deviates from this temperature by many degrees, in particular for $\rho_{2}=50 \mathrm{~cm}^{2} \mathrm{KW}^{-1}$. It is thus clear that the combined operation of two PCMs is better than non-PCMs to develop energy-free thermostats capable of maintaining a nearly constant temperature as the ambient temperature gradients change.

\section{CONCLUSIONS}

The proof of principle of a radiative thermostat has been demonstrated by exploiting the metal-insulator transition of two phase change materials with asymmetric thermal conductivities and emissivities. It has been shown that the thermostat is capable of maintaining a constant temperature equal to the transition one of the 


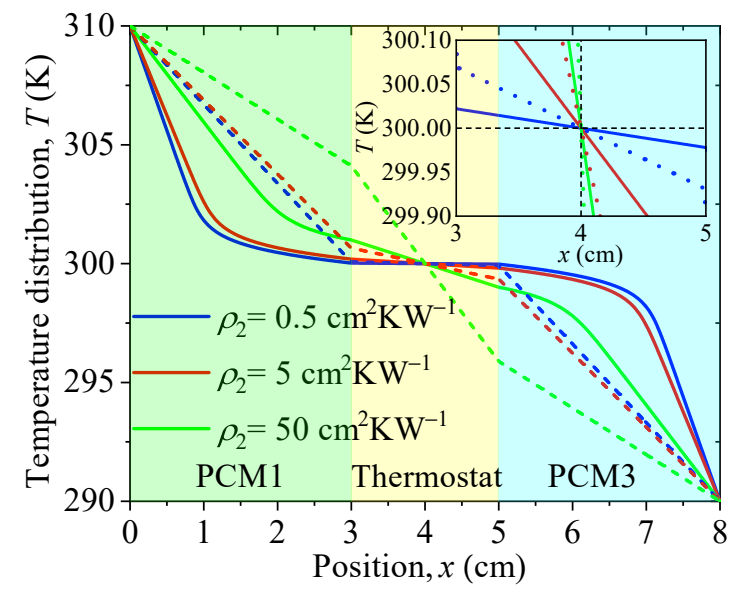

FIG. 5: Temperature profiles within the layered system shown in Fig. 1, for three values of $\rho_{2}$. The dashed lines stand for the corresponding predictions of Eq. (1) for non-PCMs with thermal conductivities $k_{n}=\bar{k}_{n}$ and emissivities $\varepsilon_{n}=\bar{\varepsilon}_{n}$. Calculations were done with $l_{2}=2$ $\mathrm{cm}, T_{h}=310 \mathrm{~K}$ and the data in caption of Fig. 3.

used phase change materials, without consuming energy despite the significant changes of the environmental temperature gradients. The obtained results can thus open new research avenues on energy-free temperature preservation, thermal insulation and phase change materials with desired thermal and optical properties.

\section{APPENDIX}

In practice, the asymmetric thermal conductivities of PCM1 and PCM3 shown in Fig. 1(a), can conveniently be implemented by means of layered structures made up of still and movable parts, as shown in Fig. 6(a). Following the experiments reported in the literature [30], the still part (bottom layer) is composed of five phosphor copper films $\left(k_{p}=54 \mathrm{Wm}^{-1} \mathrm{~K}^{-1}\right)$ separated by five silicon grease $\left(k_{s}=4 \mathrm{Wm}^{-1} \mathrm{~K}^{-1}\right)$ layers. On the other hand, the movable part (top layer) is made up of five bimetallic strips placed on top of the silicon grease layers. Each of these strips is composed of a phosphor copper film assambled with a shape memory alloy (SMA), such that their length and width are equal to the corresponding ones of the phosphor copper films. These latter films are slightly thicker than the silicon grease layers to level the bimetallic strips. The SMA is an alloy able to change its shape with temperature. In particular, a two-way SMA "remembers" two different shapes related to a phase transformation, one at low tempratures and another one at high temperatures, when it is heated up (or cooled down) through its transition temperature $T_{t}[48,49]$. Based on this fact, a two-way SMA able to tilt up for temperatures $T \ll T_{t}=300$ $\mathrm{K}$ and fully level for $T \gg T_{t}$, is chosen for builing the PCM1 and PCM3 of the proposed radiative thermostat. Taking into account that these PCMs are set at high and low temperatures (see Fig. 1), their bimetallic strips will be driven up and down with an opposite deformation, but a common transition temperature $T_{0}=T_{t}=300$ K. According to Fig. 6(a), the "experimental" thermal conductivities $k_{1}$ and $k_{3}$ of the respective PCM1 and PCM3 can then be estimated by the well-known model of thermal resistances in series, as follows

$$
\begin{aligned}
& \frac{2 N}{k_{1}}=\frac{N}{k_{p}}+\frac{n}{k_{s}}+\frac{N-n}{k_{c}}, \\
& \frac{2 N}{k_{3}}=\frac{N}{k_{p}}+\frac{n}{k_{c}}+\frac{N-n}{k_{s}},
\end{aligned}
$$

where $n=0,1, \ldots, N=5$ is the number of bimetallic strips tilted up (leveled) of PCM1 (PCM3) and $k_{c}$ is the thermal conductivity of one of these strips. Considering that a bimetallic strip is composed of a phosphor copper film attached to a SMA one of thermal conductivity $k_{S M A}$, its effective thermal conductivity is determined by the model of thermal resistance in parallel: $k_{c}=\left(l_{p} k_{p}+l_{S M A} k_{S M A}\right) /\left(l_{p}+l_{S M A}\right)$, with $l_{p}$ and $l_{S M A}$ being the heights through which the heat propagate inside the phosphor copper and SMA films, respectively. Taking into account that $k_{S M A}$ can also change with temperature [49], the condition $l_{S M A} \ll l_{p}$ is assumed, such that $k_{c} \approx k_{p}$, which is nearly independent of temperature, for the range of temperatures considered in this work. Figure 6(b) shows the "experimental" predictions (solid lines) of Eqs. (5a) and (5b) in comparison with the respective "theoretical" ones (dashed lines) predicted by Eq. (2a) for the thermal conductivities of PCM1 and PCM3. One can see that the solid curves are similar to the corresponding dashed ones describing the asymmetric temperature variations of $k_{1}$ and $k_{3}$ between 295 and $305 \mathrm{~K}$. In practice, these variations can be smoother by increasing the number $2 N$ of layers, as established by Eqs. (5a) and (5b). The proposed methodology based on a SMA could thus be implemented to observe the operation of a radiative thermostat, in a similar way as was done with the conductive one [30].
[1] L. L. N. Laboratory, "Energy flow charts: Charting the complex relationships among energy, water, and carbon," (2019: https://flowcharts.llnl.gov).
[2] E. Goldstein, A. Raman, and S. Fan, Nat. Energy 2, 17143 (2017).

[3] Y. Li, X. Shen, Z. Wu, J. Huang, Y. Chen, Y. Ni, and J. Huang, Phys. Rev. Lett. 115, 195503 (2015). 


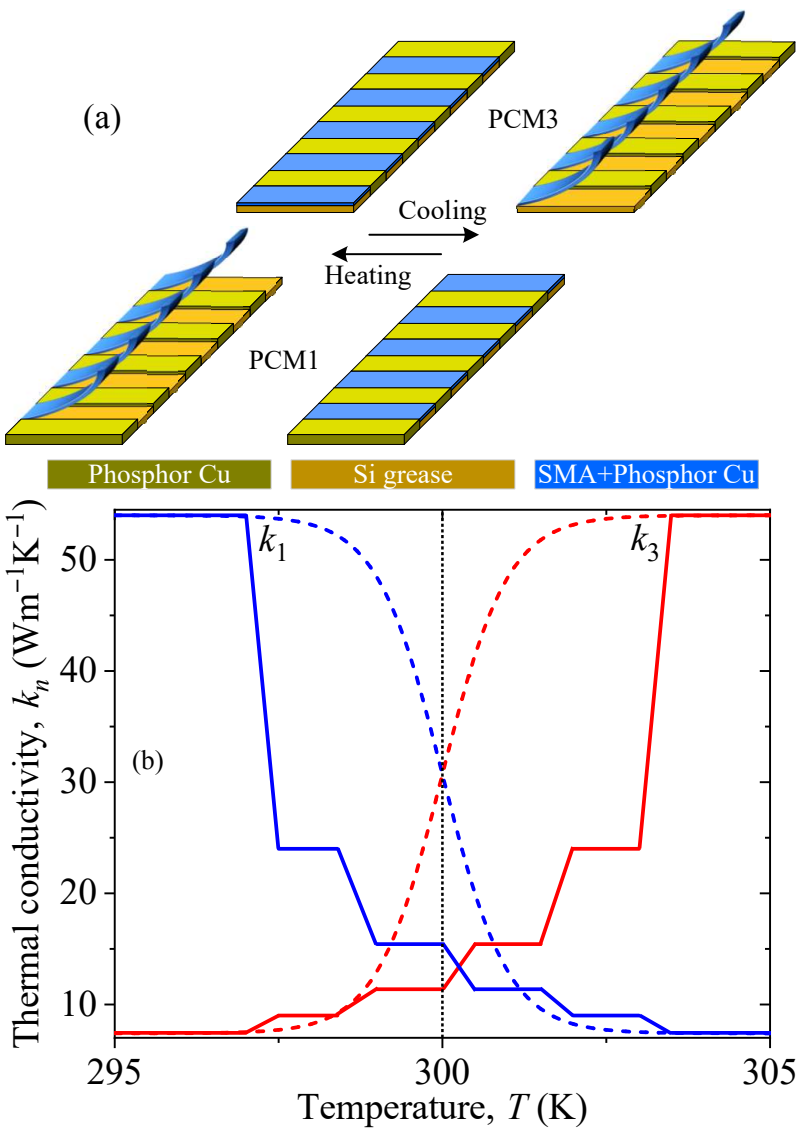

FIG. 6: (a) Schemes of the PCM1 and PCM3 composed of layered systems based on bimetallic strips. These strips tilt up for low temperatures $(T \ll 300 \mathrm{~K})$ and level for high ones $(T \gg 300 \mathrm{~K})$, due to its composition with a SMA, as demonstrated in the literature [30]. The

thermal conductivities of PCM1 and PCM3 thus depend on the shape of the bimetallic strips and their temperature dependences are shown in (b). The dashed lines stand for the predictions of Eq. (2b), while the solid ones are predicted by Eqs. (5a) and (5b), assuming the leveling of $0,1,2,3,4$, and 5 bimetallic strips. Calculations in (b) were done considering that the shape change of a strip is generated by a temperature variation of $1 \mathrm{~K}$ followed by $0.5 \mathrm{~K}$ between the transformations of two consecutive strips.

[4] B. Hu, L. Yang, and Y. Zhang, Phys. Rev. Lett. 97, 124302 (2006).

[5] C. W. Chang, D. Okawa, A. Majumdar, and A. Zettl, Science 314, 1121 (2006).

[6] J. Hu, X. Ruan, and Y. P. Chen, Nano Letters 9, 2730 (2009).

[7] N. Yang, G. Zhang, and B. Li, Appl. Phys. Lett. 95, 033107 (2009).

[8] M. Martinez-Perez, A. Fornieri, and F. Giazotto, Nat. Nanotech. 10, 303 (2009).

[9] C. R. Otey, W. T. Lau, and S. Fan, Phys. Rev. Lett. 104, 154301 (2010).
[10] S. Basu and M. Francoeur, Appl. Phys. Lett. 98, 113106 (2011).

[11] L. Zhu, C. R. Otey, and S. Fan, Appl. Phys. Lett. 100, 044104 (2012).

[12] Y. Yang, S. Basu, and L. Wang, Appl. Phys. Lett. 103, 163101 (2013).

[13] B. Li, L. Wang, and G. Casati, Phys. Rev. Lett. 93, 184301 (2004).

[14] M. Terraneo, M. Peyrard, and G. Casati, Phys. Rev. Lett. 88, 094302 (2002).

[15] D. Segal, Phys. Rev. Lett. 100, 105901 (2008).

[16] P. Ben-Abdallah and S.-A. Biehs, Appl. Phys. Lett. 103, 191907 (2013)

[17] E. Nefzaoui, J. Drevillon, Y. Ezzahri, and K. Joulain, Appl. Opt. 53, 3479 (2014).

[18] J. Ordonez-Miranda, J. M. Hill, K. Joulain, Y. Ezzahri, and J. Drevillon, J. Appl. Phys. 123, 085102 (2018).

[19] P. Ben-Abdallah and S.-A. Biehs, Phys. Rev. Lett. 112, 044301 (2014)

[20] H. Prod'homme, J. Ordonez-Miranda, Y. Ezzahri, J. Drevillon, and K. Joulain, J. Appl. Phys. 119, 194502 (2016).

[21] K. Joulain, Y. Ezzahri, J. Drevillon, and P. BenAbdallah, Appl. Phys. Lett. 106, 133505 (2015).

[22] J. Ordonez-Miranda, Y. Ezzahri, J. Drevillon, and K. Joulain, J. Appl. Phys. 119, 203105 (2016).

[23] I. Latella, O. Marconot, J. Sylvestre, L. G. Fréchette, and P. Ben-Abdallah, Phys. Rev. Applied 11, 024004 (2019).

[24] V. Kubytskyi, S.-A. Biehs, and P. Ben-Abdallah, Phys. Rev. Lett. 113, 074301 (2014).

[25] F. Yang, M. P. Gordon, and J. J. Urban, J. Appl. Phys. 125, 025109 (2019).

[26] J. Ordonez-Miranda, Y. Ezzahri, J. A. Tiburcio-Moreno, K. Joulain, and J. Drevillon, Phys. Rev. Lett. 123, 025901 (2019).

[27] K. Ito, K. Nishikawa, H. Iizuka, and H. Toshiyoshi, Appl. Phys. Lett. 105, 253503 (2014).

[28] A. Fiorino, D. Thompson, L. Zhu, R. Mittapally, S.A. Biehs, O. Bezencenet, N. El-Bondry, S. Bansropun, P. Ben-Abdallah, E. Meyhofer, and P. Reddy, ACS Nano 12, 5774 (2018).

[29] K. Ito, K. Nishikawa, and H. Iizuka, Appl. Phys. Lett. 108, 053507 (2016).

[30] X. Shen, Y. Li, C. Jiang, and J. Huang, Phys. Rev. Lett. 117, 055501 (2016).

[31] J. Wang, J. Shang, and J. Huang, Phys. Rev. Applied 11, 024053 (2019).

[32] W. J. M. Kort-Kamp, S. Kramadhati, A. K. Azad, M. T. Reiten, and D. A. R. Dalvit, ACS Photonics 5, 4554 (2018).

[33] O. Masashi, C. Kaifeng, L. Wei, and F. Shanhui, Opt. Express 26, A777 (2018).

[34] S. Wu, K. Lai, and C. Wang, Sci. Rep. 8, 7684 (2018).

[35] W. Shao-Hua, C. Mingkun, T. B. Michael, J. Vladan, H. Philip W.C., S. Luke A., and P. Michelle L., Optica 4, 1390 (2017).

[36] M. F. Modest, Radiative Heat Transfer (Academic Press, New York, 2013).

[37] F. P. Incropera, D. P. Dewitt, T. L. Bergman, and A. S. Lavine, Fundamentals of Heat and Mass Transfer (John Wiley \& Sons, Hoboken, NJ, 2011).

[38] T. Zhang and T. Luo, Small 11, 4657 (2015). 
[39] K.-K. Du, Q. Li, Y.-B. Lyu, J.-C. Ding, Y. Lu, Z.-Y. Cheng, and M. Qiu, Light Sci. Appl. 6, e16194 (2017).

[40] D.-W. Oh, C. Ko, S. Ramanathan, and D. G. Cahill, Appl. Phys. Lett. 96, 151906 (2010).

[41] J. Ordonez-Miranda, Y. Ezzahri, K. Joulain, J. Drevillon, and J. J. Alvarado-Gil, Phys. Rev. B 98, 075144 (2018).

[42] J. Ordonez-Miranda, Y. Ezzahri, J. Drevillon, and K. Joulain, Phys. Rev. Applied 6, 054003 (2016).

[43] K. Garcia-Garcia and J. Alvarez-Quintana, Int. J. Thermal Sci. 81, 76 (2014).

[44] S. Lee, K. Hippalgaonkar, F. Yang, J. Hong, C. Ko, J. Suh, K. Liu, K. Wang, J. J. Urban, X. Zhang, C. Dames, S. A. Hartnoll, O. Delaire, and J. Wu, Science 355, 371 (2017).

[45] J. A. Ramirez-Rincon, C. L. Gomez-Heredia, A. Corvisier, J. Ordonez-Miranda, T. Girardeau, F. Paumier, C. Champeaux, F. Dumas-Bouchiat, Y. Ezzahri, K. Joulain, O. Ares, and J. J. Alvarado-Gil, J. Appl. Phys. 124, 195102 (2018).

[46] C. L. Gomez-Heredia, J. A. Ramirez-Rincon, J. OrdonezMiranda, O. Ares, J. J. Alvarado-Gil, C. Champeaux,
F. Dumas-Bouchiat, Y. Ezzahri, and K. Joulain, Sci. Rep. 8, 8479 (2018).

[47] J. Yoon, H. Kim, X. Chen, N. Tamura, B. S. Mun, C. Park, and H. Ju, ACS Appl. Mater. Interfaces 8, 2280 (2016).

[48] C. Chluba, W. Ge, R. Lima de Miranda, J. Strobel, L. Kienle, E. Quandt, and M. Wuttig, Science 348, 1004 (2015).

[49] D. Dye, Nat. Mater. 14, 760 (2015).

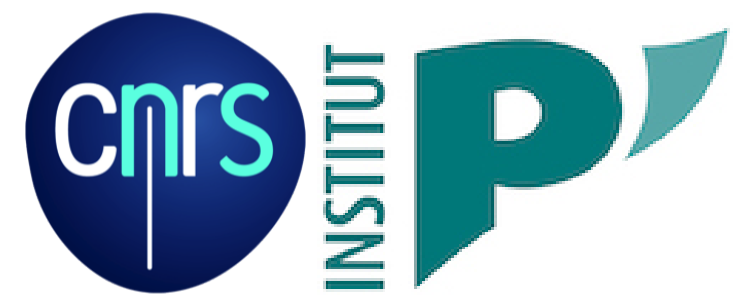

